\title{
Joint Symbol Timing and CFO Estimation for OFDM/OQAM Systems in Multipath Channels
}

\author{
Tilde Fusco (EURASIP Member), ${ }^{1}$ Angelo Petrella, ${ }^{2}$ and Mario Tanda ${ }^{3}$ \\ ${ }^{1}$ Communications Regulatory Authority, Department for Studies, Research and Education, Centro Direzionale, \\ Isola B5, 80143 Napoli, Italy \\ ${ }^{2}$ Selex Sistemi Integrati, Via Giulio Cesare 268, Bacoli, 80070 Napoli, Italy \\ ${ }^{3}$ Dipartimento di Ingegneria Biomedica, Elettronica e delle Telecomunicazioni, Università di Napoli Federico II, \\ Via Claudio 21, 80125 Napoli, Italy
}

Correspondence should be addressed to Mario Tanda, mario.tanda@unina.it

Received 27 May 2009; Revised 27 September 2009; Accepted 13 November 2009

Academic Editor: Faouzi Bader

Copyright (C) 2010 Tilde Fusco et al. This is an open access article distributed under the Creative Commons Attribution License, which permits unrestricted use, distribution, and reproduction in any medium, provided the original work is properly cited.

The problem of data-aided synchronization for orthogonal frequency division multiplexing (OFDM) systems based on offset quadrature amplitude modulation (OQAM) in multipath channels is considered. In particular, the joint maximum-likelihood (ML) estimator for carrier-frequency offset (CFO), amplitudes, phases, and delays, exploiting a short known preamble, is derived. The ML estimators for phases and amplitudes are in closed form. Moreover, under the assumption that the CFO is sufficiently small, a closed form approximate ML (AML) CFO estimator is obtained. By exploiting the obtained closed form solutions a cost function whose peaks provide an estimate of the delays is derived. In particular, the symbol timing (i.e., the delay of the first multipath component) is obtained by considering the smallest estimated delay. The performance of the proposed joint AML estimator is assessed via computer simulations and compared with that achieved by the joint AML estimator designed for AWGN channel and that achieved by a previously derived joint estimator for OFDM systems.

\section{Introduction}

In the last years, the interest for filter-bank multicarrier (FBMC) systems is increased, since they provide high spectral containment. Therefore, they have been taken into account for high-data-rate transmissions over both wired and wireless frequency-selective channels. Moreover, they have been considered for the physical layer of cognitive radio systems [1]. One of the most famous multicarrier modulation techniques is orthogonal frequency division multiplexing (OFDM), embedded in several standards such as digital audio and video broadcasting or Wi-Fi wireless LANs IEEE 802.11a/g. Other known types of FBMC systems are Filtered Multitone (FMT) systems, that have been proposed for very high-speed digital subscriber line standards [2] and are under investigation also for broadband wireless applications [3] and, moreover, OFDM based on offset QAM modulation (OQAM), considered by the 3GPP standardization forum for improved down-link UTRAN interfaces [4].
Unlike OFDM, OFDM/OQAM systems do not require the presence of a cyclic prefix (CP) in order to combat the effects of frequency selective channels. The absence of the $\mathrm{CP}$ implies on one hand the maximum spectral efficiency and, on the other hand, an increased computational complexity. However, since the subchannel filters are obtained by complex modulation of a single filter, efficient polyphase implementations are possible. Another fundamental difference between OFDM and OFDM/OQAM systems is the adoption in the latter case of pulse shaping filters very well localized in time and frequency $[5,6]$.

OFDM/OQAM systems are more sensitive to synchronization errors than single-carrier systems. In particular, carrier frequency-offset (CFO) and symbol timing (ST) estimation errors can lead to a performance degradation. For this reason, it is very important to derive efficient synchronization schemes. In the last years several studies have been focused on blind or data-aided synchronization for OFDM/OQAM systems. For example, in $[7,8]$ blind CFO estimators have been derived. Moreover, in [9] a blind 
joint CFO and ST estimator is proposed. Furthermore, in [10] a synchronization scheme for data-aided ST and CFO estimation with robust acquisition properties in dispersive channels is developed. Finally, in $[11,12]$ a full synchronization method utilizing frequency domain scattered pilots in the time domain is proposed. However, all cited estimators are designed for down-link communications.

In this paper we consider the problem of data-aided synchronization for OFDM/OQAM systems in multipath channels. In particular, the joint maximum-likelihood (ML) estimator for $\mathrm{CFO}$, amplitudes, phases, and delays, exploiting a short known preamble, is derived. The ML estimators for phases and amplitudes are in closed form. Moreover, under the assumption that the CFO is sufficiently small, a closed form approximate ML (AML) CFO estimator is obtained. By exploiting the obtained closed form solutions a cost function whose peaks provide an estimate of the delays is derived. In particular, the ST (i.e., the delay of the first multipath component) is obtained by considering the smallest estimated delay. The proposed joint estimator is derived with reference to a down-link scenario; however, by following an approach similar to that considered in [13], it can be easily modified to be exploited for up-link communications. The performance of the proposed joint AML estimator is assessed via computer simulations and compared with that achieved by the joint AML estimator designed for AWGN channel and that achieved by a previously derived joint estimator for OFDM systems. The paper is organized as follows. In Section 2 the OFDM/OQAM system model is described. In Section 3 the proposed data-aided estimator is described. In Section 4 numerical results obtained in AWGN and multipath channel are presented and discussed. Finally, conclusions are drawn in Section 5.

Notation $1 . j \triangleq \sqrt{-1}$, superscript $(\cdot)^{*}$ denotes the complex conjugation, $\mathfrak{R}[\cdot]$ real part, $\mathfrak{I}[\cdot]$ imaginary part, and $|\cdot|$ absolute value. Moreover, $(\cdot)^{T}$ denotes transpose and $\angle[\cdot]$ the argument of a complex number in $[-\pi, \pi)$. Finally, lower case boldface symbols denote column vectors.

\section{System Model}

Let us consider an OFDM/OQAM system with $N$ subcarriers in a multipath channel. The received signal, in the presence of a CFO normalized to subcarrier spacing $\varepsilon=\Delta f T$, can be written as

$$
r(t)=e^{j(2 \pi / T) \varepsilon t} \sum_{i=1}^{N_{c}} \gamma_{i} e^{j \phi_{i}} \mathcal{S}\left(t-\tau_{i}\right)+n(t)
$$

where $s(t)$ is the information-bearing signal, $N_{c}$ is the number of multipath components, and, $\gamma_{i}, \phi_{i}$, and $\tau_{i}$ denote amplitude, phase, and delay, respectively, of the ith path. Moreover, in (1) $n(t)$ is a zero-mean complex-valued white Gaussian noise process with independent real and imaginary part, each with two-sided power spectral density $\sigma_{n}^{2} / 2$. The received signal $r(t)$ is filtered with an ideal lowpass filter with a bandwidth of $1 / T_{s}$, where $T_{s}$ denotes the sampling period. The sampled signal $s\left(k T_{s}\right)$ is equal to

$$
\begin{aligned}
s\left(k T_{s}\right)= & \sqrt{\frac{N}{2 N_{u}}} \sum_{p=0}^{S-1} \sum_{l \in \mathcal{A}} e^{j l((2 \pi / N) k+\pi / 2)} \\
& \times\left[a_{p, l}^{R} g\left(k T_{s}-p T\right)+j a_{p, l}^{I} g\left(k T_{s}-p T-\frac{T}{2}\right)\right],
\end{aligned}
$$

where $T=N T_{s}$ is the OFDM/OQAM symbol interval and $S$ denotes the number of information-bearing symbols in the burst. Moreover, in (2) $\mathcal{A}$ is the set of size $N_{u}$ of used subcarriers, $a_{p, l}^{R}$ and $a_{p, l}^{I}$ denote the real and imaginary part of the complex data symbol transmitted on the $l$ th subcarrier during the $p$ th OFDM/OQAM symbol, while the real-valued and unit-energy pulse-shaping filter $g(t)$ is bandlimited within $[-1 / T, 1 / T]$.

\section{Joint Symbol Timing and CFO Estimator}

In this section we consider the problem of data-aided synchronization for OFDM/OQAM systems in multipath channels. In particular, we derive the joint ML estimator for CFO, amplitudes, phases, and delays, exploiting a short known preamble embedded in the received burst. Specifically, the known preamble is given by

$$
\begin{aligned}
z\left(k T_{s}\right)= & \sqrt{\frac{N}{2 N_{u}}} \sum_{p=0}^{L-1} \sum_{l \in \mathcal{P}} e^{j l\left((2 \pi / T) k T_{s}+\pi / 2\right)} \\
& \times\left[a_{p, l}^{R} g\left(k T_{s}-p T\right)+j a_{p, l}^{I} g\left(k T_{s}-p T-\frac{T}{2}\right)\right],
\end{aligned}
$$

where $L$ is the number of OFDM/OQAM symbols in the preamble, $\mathcal{P}$ is the set of pilot subcarriers, and $a_{p, l}^{R}$, $a_{p, l}^{I} \in\{-1,1\}, 0 \leq p \leq L-1, l \in \mathcal{P}$, denote the known pilot symbols. Note that the duration of the preamble is $D=(\beta+1 / 2+L-1) T$ where $\beta$ is the overlap parameter, that is, the ratio between the length of the truncated pulseshaping filter and the OFDM/OQAM symbol interval $T$. By considering an observations window of total length $\eta N$ containing the nonzero support of the received preamble, the likelihood function for the unknown parameters $\varepsilon$, and, $\gamma_{i}$, $\phi_{i}$, and $\tau_{i}, i=1, \ldots, N_{c}$, is given by

$$
\begin{aligned}
& \Lambda(\tilde{\gamma}, \tilde{\phi}, \tilde{\boldsymbol{\tau}}, \tilde{\varepsilon}) \\
& \quad=\exp \left\{-\frac{T_{s}}{\sigma_{n}^{2}} \sum_{k=0}^{n N-1}\left|r\left(k T_{s}\right)-\sum_{i=1}^{N_{c}} \tilde{\gamma}_{i} e^{j \tilde{\phi}_{i}} z^{\tau_{i}, \tilde{\varepsilon}}\left(k T_{s}\right)\right|^{2}\right\},
\end{aligned}
$$

where $\tilde{\boldsymbol{\gamma}}=\left[\tilde{\gamma}_{1}, \tilde{\gamma}_{2}, \ldots, \tilde{\gamma}_{N_{c}}\right]^{T}, \tilde{\boldsymbol{\phi}}=\left[\tilde{\phi}_{1}, \tilde{\phi}_{2}, \ldots, \tilde{\phi}_{N_{c}}\right]^{T}, \tilde{\boldsymbol{\tau}}=$ $\left[\tilde{\tau}_{1}, \tilde{\tau}_{2}, \ldots, \tilde{\tau}_{N_{c}}\right]^{T}$,

$$
z^{\tau, \varepsilon}\left(k T_{s}\right) \triangleq z\left(k T_{s}-\tau\right) e^{j(2 \pi / N) \varepsilon k},
$$


and the notation of the type $\tilde{x}$ indicates trial value of $x$. Thus, the log-likelihood function for the parameters of interest results to be (up to irrelevant factors)

$$
\begin{aligned}
\ln \Lambda(\tilde{\boldsymbol{\gamma}}, \tilde{\phi}, \tilde{\boldsymbol{\tau}}, \tilde{\varepsilon})= & -\sum_{k=0}^{\eta N-1}\left|\sum_{i=1}^{N_{c}} \tilde{\gamma}_{i} e^{j \tilde{\phi}_{i}} z^{\tilde{\tau}_{i}, \tilde{\varepsilon}}\left(k T_{s}\right)\right|^{2} \\
& +2 \Re\left[\sum_{k=0}^{\eta N-1} r\left(k T_{s}\right) \sum_{i=1}^{N_{c}} \tilde{\gamma}_{i} e^{-j \tilde{\phi}_{i}} z^{\tilde{\tau}_{i}, \tilde{\varepsilon}}\left(k T_{s}\right)^{*}\right] .
\end{aligned}
$$

The first term in the right-hand side (RHS) of (6) for $N \gg 1$ can be approximated as

$$
\sum_{k=0}^{\eta N-1}\left|\sum_{i=1}^{N_{c}} \tilde{\gamma}_{i} e^{j \tilde{\phi}_{i}} z^{\tilde{\tau}_{i}, \tilde{\varepsilon}}\left(k T_{s}\right)\right|^{2} \simeq \sum_{i=1}^{N_{c}} \tilde{\gamma}_{i}^{2} \sum_{k=0}^{\eta N-1}\left|z\left(k T_{s}-\tilde{\tau}_{i}\right)\right|^{2} .
$$

Therefore, the log-likelihood function can be written as

$$
\ln \Lambda(\tilde{\gamma}, \tilde{\phi}, \tilde{\boldsymbol{\tau}}, \widetilde{\mathcal{\varepsilon}})=\sum_{i=1}^{N_{c}}\left\{\tilde{\gamma}_{i} \Re\left[e^{-j \tilde{\phi}_{i}} c\left(\tilde{\tau}_{i}, \widetilde{\mathcal{\varepsilon}}\right)\right]-\tilde{\gamma}_{i}^{2} d\left(\tilde{\tau}_{i}\right)\right\}
$$

where

$$
\begin{gathered}
d(\tilde{\tau})=\sum_{k=0}^{\eta N-1}\left|z\left(k T_{s}-\tilde{\tau}\right)\right|^{2}, \\
c(\tilde{\tau}, \widetilde{\mathcal{\varepsilon}}) \triangleq \sum_{l \in \mathcal{P}} e^{-j(\pi / 2) l} \sum_{p=0}^{L-1}\left[a_{p, l}^{R} w_{p}^{(l)}(\tilde{\tau}, \widetilde{\varepsilon})-j a_{p, l}^{I} \widehat{w}_{p}^{(l)}(\tilde{\tau}, \widetilde{\mathcal{\varepsilon}})\right]
\end{gathered}
$$

with

$$
\begin{aligned}
w_{p}^{(l)}(\tilde{\tau}, \widetilde{\mathcal{E}}) \triangleq & \sqrt{\frac{2 N}{N_{u}}} e^{j(2 \pi / T) \tilde{\tau} l} \sum_{k=0}^{\eta N-1} r\left(k T_{s}\right) \\
& \times g\left(k T_{s}-p T-\tilde{\tau}\right) e^{-j(2 \pi / N) k(l+\tilde{\varepsilon})}, \\
\hat{w}_{p}^{(l)}(\tilde{\tau}, \tilde{\mathcal{E}}) \triangleq & w_{p}^{(l)}\left(\tilde{\tau}+\frac{T}{2}, \tilde{\varepsilon}\right) e^{-j \pi l} .
\end{aligned}
$$

From (8), it immediately follows that the ML estimator for phase and amplitude of the $i$ th path is given by

$$
\begin{aligned}
& \hat{\phi}_{i_{M L}}\left(\tilde{\tau}_{i}, \tilde{\mathcal{\varepsilon}}\right)=\arg \max _{\widetilde{\phi}_{i}}\{\ln \Lambda(\tilde{\gamma}, \tilde{\phi}, \tilde{\boldsymbol{\tau}}, \tilde{\mathcal{\varepsilon}})\}=\angle c\left(\tilde{\tau}_{i}, \tilde{\mathcal{\varepsilon}}\right), \\
& \hat{\gamma}_{i_{M L}}\left(\tilde{\tau}_{i}, \tilde{\mathcal{\varepsilon}}\right)=\arg \max _{\widetilde{\gamma}_{i}}\{\ln \Lambda(\tilde{\gamma}, \tilde{\phi}, \tilde{\tau}, \tilde{\varepsilon})\}=\frac{\left|c\left(\tilde{\tau}_{i}, \tilde{\mathcal{\varepsilon}}\right)\right|}{2 d\left(\tilde{\tau}_{i}\right)} .
\end{aligned}
$$

Moreover, by replacing the estimate of the phase and the amplitude of each path in (8) we get

$$
\ln \Lambda(\hat{\boldsymbol{\gamma}}(\tilde{\boldsymbol{\tau}}, \widetilde{\varepsilon}), \hat{\phi}(\tilde{\boldsymbol{\tau}}, \widetilde{\varepsilon}), \tilde{\boldsymbol{\tau}}, \widetilde{\varepsilon})=\sum_{i=1}^{N_{c}} \frac{\left|c\left(\tilde{\tau}_{i}, \widetilde{\varepsilon}\right)\right|^{2}}{2 d\left(\tilde{\tau}_{i}\right)}
$$

Therefore, the joint estimatorfor CFO and delays is given by

$$
\left(\widehat{\boldsymbol{\tau}}_{M L}, \widehat{\varepsilon}_{M L}\right)=\arg \max _{(\widetilde{\boldsymbol{\tau}}, \widetilde{\varepsilon})}\left\{\sum_{i=1}^{N_{c}} \frac{\left|c\left(\tilde{\tau}_{i}, \widetilde{\mathcal{E}}\right)\right|^{2}}{2 d\left(\tilde{\tau}_{i}\right)}\right\} .
$$

The derived joint ML estimator evaluates, for each trial value of each delay $\tilde{\tau}_{i}, i=1,2, \ldots, N_{c}$, and for each trial value of the CFO $\widetilde{\mathcal{E}}$, the response of the filter matched to the pulse shaping filter $g(\cdot)$ to the CFO compensated and downconverted signal $r\left(k T_{s}\right) e^{-j(2 \pi / N) \tilde{\varepsilon} k} e^{-j(2 \pi / N) k l}$ at the time instants $\tilde{\tau}_{i}+p T$ and $\tilde{\tau}_{i}+p T+T / 2,0 \leq p \leq L-1$. Specifically, the downconversion is performed by considering all the frequencies of the pilot subcarriers. Then, exploiting the known pilot symbols, these quantities are combined according to (11) (or (12)), (10), and (16). Note that the proposed algorithm can be exploited also in the up-link to estimate the desired parameters by considering only the subcarriers assigned to the user of interest. The joint ML estimate for delays and $\mathrm{CFO}$ is obtained by considering the value of $(\tilde{\boldsymbol{\tau}}, \widetilde{\mathcal{\varepsilon}})$ that maximizes the statistic in (15). The $\left(N_{c}+1\right)$-dimensional maximization required by the joint ML estimator in (16) undertakes heavy computational burden. Therefore, in the following is derived a more feasible synchronization scheme by exploiting the assumption that the CFO is sufficiently small. Specifically, taking into account that the observations window contains the nonzero support of the received preamble and that the prototype filter $g\left(k T_{s}\right)$ is different from zero for $k \in\{0, \beta N-1\}$, from (11) it follows that

$$
\begin{aligned}
w_{p}^{(l)}(\tilde{\tau}, \tilde{\varepsilon})= & \sqrt{\frac{2 N}{N_{u}}} e^{-j 2 \pi \tilde{\varepsilon} p} e^{-j(2 \pi / N) \tilde{\varepsilon} \tilde{\theta}} \\
& \times \sum_{m=0}^{\beta N-1} r\left[(m+p N+\tilde{\theta}) T_{s}\right] \times e^{-j(2 \pi / N) \tilde{\varepsilon} m} \\
& \times g\left(m T_{s}\right) e^{-j(2 \pi / N) m l},
\end{aligned}
$$

where the integer $\tilde{\theta}$ is the trial value of the delay $\tilde{\tau}$ normalized to the sampling interval, $\tilde{\theta}=\tilde{\tau} / T_{s}$. Then, under the assumption that the CFO is sufficiently small within a time $\Delta Q$ comparable with the length of the prototype filter $e^{-j(2 \pi / N) \tilde{\varepsilon} \Delta Q} \simeq 1$, it results that

$$
\begin{gathered}
w_{p}^{(l)}(\tilde{\tau}, \widetilde{\varepsilon}) \simeq e^{-j 2 \pi \tilde{\varepsilon} p} e^{-j(2 \pi / N) \tilde{\varepsilon} \tilde{\theta}} u_{l}(p N+\tilde{\theta}) \\
\widehat{w}_{p}^{(l)}(\tilde{\tau}, \widetilde{\varepsilon}) \simeq e^{-j 2 \pi \tilde{\varepsilon}(p+(1 / 2))} e^{-j(2 \pi / N) \tilde{\varepsilon} \tilde{\theta}} e^{-j \pi l} u_{l}\left(p N+\frac{N}{2}+\tilde{\theta}\right),
\end{gathered}
$$

where

$$
u_{l}(k) \triangleq \sum_{m=0}^{\beta N-1} r\left[(m+k) T_{s}\right] g\left(m T_{s}\right) e^{-j(2 \pi / N) m l} .
$$


In particular, into the case of a training sequence composed of $L=1$ OFDM/OQAM symbol, (10), taking into account (18)-(20), becomes

$$
\begin{aligned}
c(\tilde{\tau}, \widetilde{\mathcal{E}}) & =\sum_{l \in \mathcal{P}} e^{-j(\pi / 2) l}\left[a_{0, l}^{R} w_{0}^{(l)}(\tilde{\tau}, \widetilde{\mathcal{E}})-j a_{0, l}^{I} \widehat{w}_{0}^{(l)}(\tilde{\tau}, \tilde{\mathcal{\varepsilon}})\right] \\
& \simeq A(\tilde{\tau})+e^{-j \pi \tilde{\varepsilon}} B(\tilde{\tau})
\end{aligned}
$$

with

$$
\begin{gathered}
A(\tilde{\tau}) \triangleq e^{-j(2 \pi / N) \tilde{\varepsilon} \tilde{\theta}} \sum_{l \in \mathcal{P}} e^{-j(\pi / 2) l} a_{0, l}^{R} u_{l}(\tilde{\theta}), \\
B(\tilde{\tau}) \triangleq e^{-j(2 \pi / N) \tilde{\varepsilon} \tilde{\theta}} \sum_{l \in \mathcal{P}} e^{-j(\pi / 2)(l+1)} a_{0, l}^{I} e^{-j \pi l} u_{l}(N / 2+\tilde{\theta}) .
\end{gathered}
$$

Therefore, under the assumption $|\varepsilon| \ll N / \Delta Q$ and in the case of a training sequence with $L=1$ OFDM/OQAM symbol, the AML estimator for CFO, taking into account (16), is given by

$$
\begin{aligned}
\widehat{\mathcal{\varepsilon}}_{\mathrm{AML}}(\tilde{\boldsymbol{\tau}}) & =\arg \max _{\widetilde{\varepsilon}}\left\{\sum_{i=1}^{N_{c}} \frac{\left|A\left(\tilde{\tau}_{i}\right)+e^{-j \pi \tilde{\varepsilon}} B\left(\tilde{\tau}_{i}\right)\right|^{2}}{2 d\left(\tilde{\tau}_{i}\right)}\right\} \\
& =\frac{1}{\pi} \angle\left\{\sum_{i=1}^{N_{c}} \frac{A^{*}\left(\tilde{\tau}_{i}\right) B\left(\tilde{\tau}_{i}\right)}{d\left(\tilde{\tau}_{i}\right)}\right\} .
\end{aligned}
$$

Moreover, from (16) and (21)-(24) it follows that

$$
\begin{aligned}
\hat{\boldsymbol{\tau}}_{\mathrm{AML}}=\arg \max _{\widetilde{\tau}}\left\{\sum_{i=1}^{N_{c}}\left[\frac{\left|A\left(\tilde{\tau}_{i}\right)\right|^{2}+\left|B\left(\tilde{\tau}_{i}\right)\right|^{2}}{2 d\left(\tilde{\tau}_{i}\right)}\right]\right. \\
\left.+\left|\sum_{i=1}^{N_{c}} \frac{A^{*}\left(\tilde{\tau}_{i}\right) B\left(\tilde{\tau}_{i}\right)}{d\left(\tilde{\tau}_{i}\right)}\right|\right\} .
\end{aligned}
$$

Thus, although under the assumption of small CFO values a closed form approximate estimator can be obtained, the estimation of the delays remains an $N_{c}$-dimensional problem. To simplify the estimation of the delays the last term in the RHS of (25) can be neglected, and, then, we obtain

$$
\hat{\boldsymbol{\tau}}_{\mathrm{AML} 1}=\arg \max _{\tilde{\boldsymbol{\tau}}}\left\{\sum_{i=1}^{N_{c}} \frac{\left|A\left(\tilde{\tau}_{i}\right)\right|^{2}+\left|B\left(\tilde{\tau}_{i}\right)\right|^{2}}{2 d\left(\tilde{\tau}_{i}\right)}\right\} .
$$

In this case, if the number of paths $N_{c}$ is known, it immediately follows that the $N_{c}$-dimensional vector of delays that maximize (26) can be obtained by considering the $N_{c}$ points where the cost function

$$
\operatorname{MD}(\tilde{\tau})=\frac{|A(\tilde{\tau})|^{2}+|B(\tilde{\tau})|^{2}}{d(\tilde{\tau})}
$$

presents the highest $N_{c}$ peaks. Moreover, the lowest among the obtained delays represents an estimate of the ST. If the number of paths $N_{c}$ is not known in advance, a sufficiently high number of paths should be considered to avoid to lose a strong path and, moreover, to avoid to consider very weak paths. The obtained delays can be substituted in (24) to obtain the CFO estimate, and, finally, phases and amplitudes can be obtained from (13) and (14), respectively. Note that the numerical results reported in the next section show that the considered approximation of (25) leads to a symbol timing estimator with satisfactory performance if the number of subcarriers is sufficiently large.

In Appendix A we analyzed the accuracy of the closed form AML CFO estimator (24) in the absence of noise in a single-path channel and in the case of perfect ST synchronization $\left(N_{c}=1\right.$ and $\tau_{1}=0$ in (1)). Specifically, it is shown that in this case also if the interference from the data burst following the training symbol is neglected, the CFO estimator is expected to exhibit a performance floor. However, it is shown in the next section that this performance floor can be substantially reduced if the training symbol satisfies the condition

$$
\sum_{l \in \mathcal{P}} a_{0, l}^{R} a_{0, l}^{I}=0
$$

Moreover, in Appendix B is derived an approximate expression for the mean square error (MSE) of the AML CFO estimator in (24) for a single-path channel and in the case of perfect ST synchronization. In particular, in Appendix B it is shown that in this case the MSE can be approximated by

$$
E\left[(\widehat{\varepsilon}-\varepsilon)^{2}\right]=\frac{2}{\pi^{2} N S N R} \frac{1}{\left|\sum_{k=0}^{\beta N-1} \bar{g}(k)^{2} e^{j(2 \pi / N) \varepsilon k}\right|^{2}},
$$

where $\mathrm{SNR} \triangleq \gamma^{2} / \sigma_{n}^{2}$ and $\bar{g}(k) \triangleq g\left(k T_{s}\right) / \sqrt{\sum_{l=0}^{\beta N-1} g^{2}\left(l T_{s}\right)}$. Note that for $\varepsilon=0$ the MSE in (29) is coincident with the MSE of the CFO estimator for OFDM systems proposed by Schmidl and Cox (SC) in [14]. It is worthwhile to emphasize that the MSE in (29) has been derived by neglecting the interference at the output of each matched filter due to adjacent subcarriers. Therefore, the actual performance of the proposed AML CFO estimator presents a floor that is not predicted by (29). However, it is shown in the next section that the approximate expression in (29) can be exploited in the range of moderate SNR values.

\section{Numerical Results and Comparisons}

In this section the performance of the proposed joint AML estimator is assessed via computer simulations. A number of 5000 Monte Carlo trials has been performed under the following conditions (unless otherwise stated):

(1) the considered OFDM/OQAM system has a bandwidth $B=1 / T_{s}=11.2 \mathrm{MHz}$;

(2) the data symbols $a_{p, l}^{R}$ and $a_{p, l}^{I}$ are the real and imaginary part of QPSK symbols;

(3) the length of the considered prototype filter (designed with the frequency sampling technique [15]) is $L P=\beta N$, where the overlap parameter $\beta$ is fixed at $\beta=4$; 


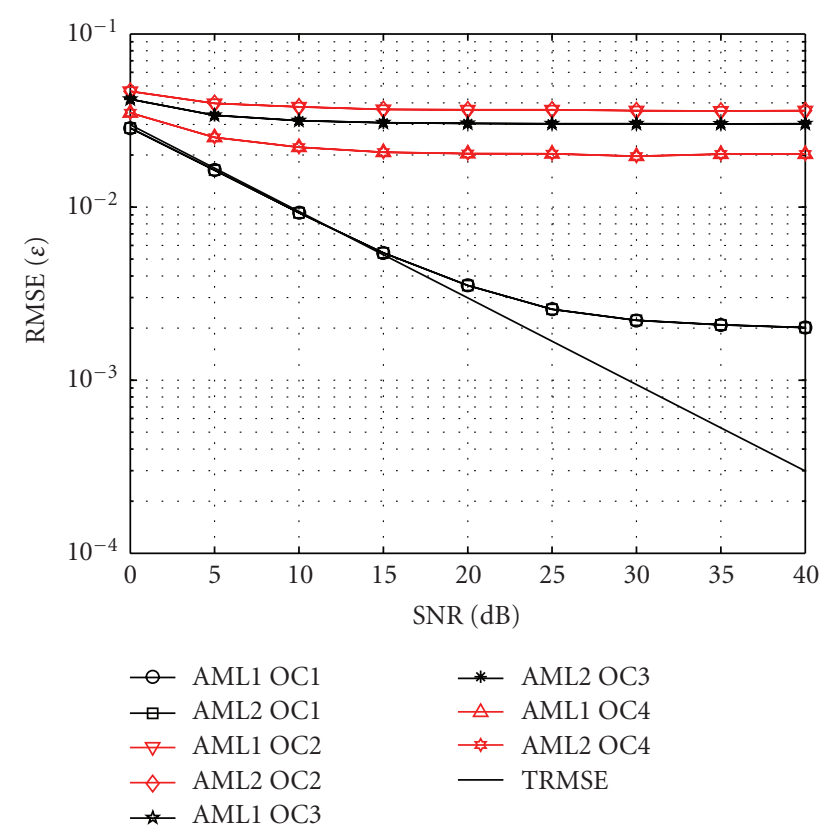

FIGURe 1: Performance of the proposed AML CFO estimators in AWGN channel.

(4) the considered multipath fading channel model is the ITU Vehicular A [16], which has six multipaths with differential delays $0,0.31,0.71,1.09$, 1.73 , and 2.51 microseconds and relative powers $0,-1,-9,-10,-15$, and $-20 \mathrm{~dB}$;

(5) the channel is fixed in each run but it is independent from one run to another.

In the first set of simulations we have tested the sensitivity of the performance of the derived CFO estimators to the condition (28) and to the interference due to the data burst sent after the training symbol. Specifically, four operating conditions have been considered:

(1) in the first case, denoted as OC1, condition (28) is satisfied and, moreover, to reduce the interference due to the data symbols, the useful data in the whole burst is delayed with respect to the preamble of the burst by one OFDM/OQAM symbol interval;

(2) in the second case, denoted as OC2, condition (28) is not satisfied and the data burst is not delayed;

(3) in the third case, termed OC3, condition (28) is not satisfied and the data burst is delayed;

(4) in the fourth case, termed OC4, condition (28) is satisfied and the data burst is not delayed.

Figures 1 and 2 display the root mean square error (RMSE) of the considered CFO estimators as a function of SNR in the previously described operating conditions and in the case where the number of subcarriers is $N=256$ and the actual value of the normalized CFO is $\varepsilon=0.2$. Specifically, the AML CFO estimator for multipath channel

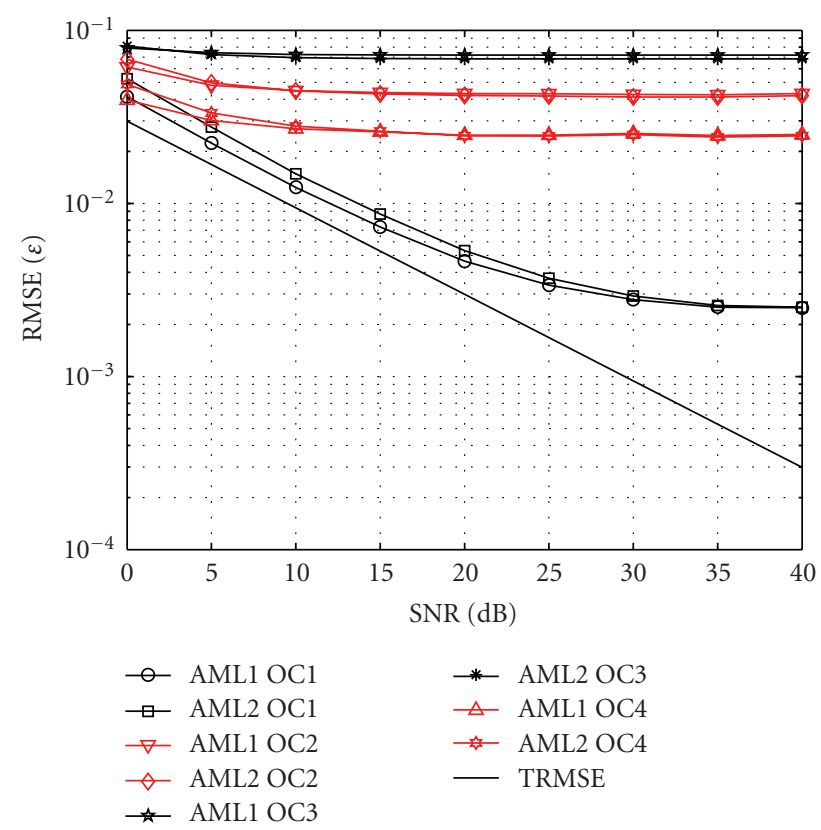

FIGURe 2: Performance of the proposed AML CFO estimators in ITU Vehicular A multipath channel.

reported in (24) is denoted as AML1 while the label AML2 indicates the AML estimator for AWGN channel, that is, that based on the choice $N_{c}=1$. In the case of the AML1 CFO estimator two paths have been considered to avoid to lose a strong path and, moreover, to avoid to consider very weak paths. As one would expect, the performance of both AML1 and AML2 estimators is coincident in AWGN channel (see Figure 1) while the AML1 outperforms the AML2 estimator in multipath channel (see Figure 2). Moreover, only when condition (28) is satisfied (curves labeled as OC1 and OC4), the insertion of the considered delay in the data burst can lead to a significant performance improvement both in AWGN and multipath channel. In particular, when condition (28) is satisfied and the data burst is delayed (curves labeled as OC1), a floor is observed only around $\mathrm{SNR}=30 \mathrm{~dB}$. In Figures 1 and 2 is also reported the theoretical RMSE (TRMSE) predicted by (29). The results show that the derived expression can be exploited in AWGN channel for $\mathrm{SNR} \leq 15 \mathrm{~dB}$. As regards the performance of the AML1 and AML2 ST estimators no errors were observed in AWGN channel while in multipath channel A an RMSE (normalized to the OFDM/OQAM interval $T$ ) less than $3 \cdot 10^{-3}$ was observed in all operating conditions for $\mathrm{SNR} \geq 5 \mathrm{~dB}$. Taking into account the previous results in the following experiments only the operating condition OC1 is considered since it assures the best performance. In particular, in Figures 3 and 4 the normalized RMSE of the AML CFO estimators is compared with that of the SC estimator proposed in [14], both in AWGN (Figure 3) and in multipath channel A (Figure 4). Specifically, the number of subcarriers is $N=256$ and the actual value of the normalized $\mathrm{CFO}$ is $\varepsilon=0.2$. Note that the performance comparison with the SC estimator is made by exploiting 


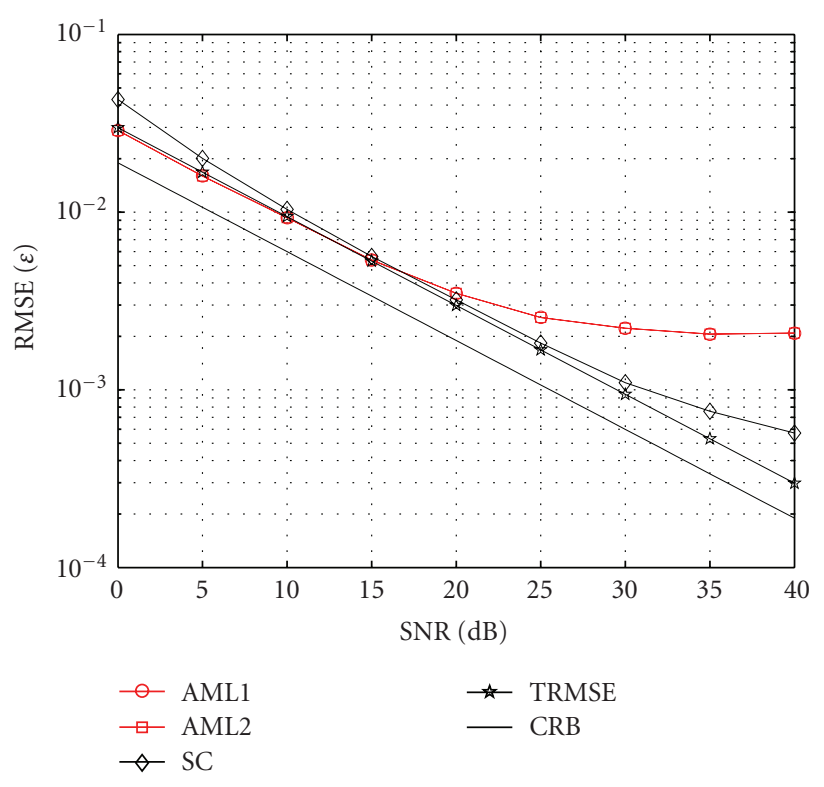

FIGURE 3: Performance of the considered CFO estimators in AWGN channel.

the proposed algorithm in an OFDM/OQAM system and the SC algorithm in an OFDM system, and, moreover, in the case of the SC algorithm an OFDM symbol with two equal parts is exploited. The results show that in multipath channel A the performance of both estimators presents a floor, but for different reasons. In the case of the SC estimator the floor is due to the inaccuracy in the ST estimate (normalized RMSE nearly equal to $2 \cdot 10^{-1}$ (see Figure 5 )), while in the case of the AML estimator the floor is due (as well as in the AWGN channel (see Figure 3)) to the interference from adjacent subcarriers. Moreover, in Figures 3 and 4 is reported also the Cramèr -Rao bound (CRB) on CFO estimation for OFDM/OQAM systems. The performance loss with respect to the $\mathrm{CRB}$ is quite contained for SNR values lower than $20 \mathrm{~dB}$. Specifically, as it is shown in the following the accuracy is sufficient to assure a negligible degradation with respect to the case of perfect synchronization.

To gain some insight about the acquisition range of the considered estimators in Figure 6 is reported the normalized RMSE of the AML CFO estimators as a function of the actual value of the normalized CFO $\varepsilon$ in AWGN (solid lines) and multipath channel A (dashed lines) for SNR $=10 \mathrm{~dB}$. In Figure 6 is also reported the RMSE of the SC estimator. Note that in this case a number of 10000 Monte Carlo trials have been performed. The results show that although the AML estimators have been derived under the assumption of small values of CFO, they assure a satisfactory performance in the range $\varepsilon \in[-0.8,0.8]$. Of course if the value of the normalized CFO can belong to a larger interval, an additional stage at the beginning of the preamble needs to be inserted to obtain a coarse estimate of the CFO within a sufficiently wide range.

Figures 7 and 8 show the normalized RMSE of the considered CFO estimators as a function of the number of subcarriers $N$ in AWGN (Figure 7) and in multipath channel A (Figure 8), and for two SNR conditions. The results show

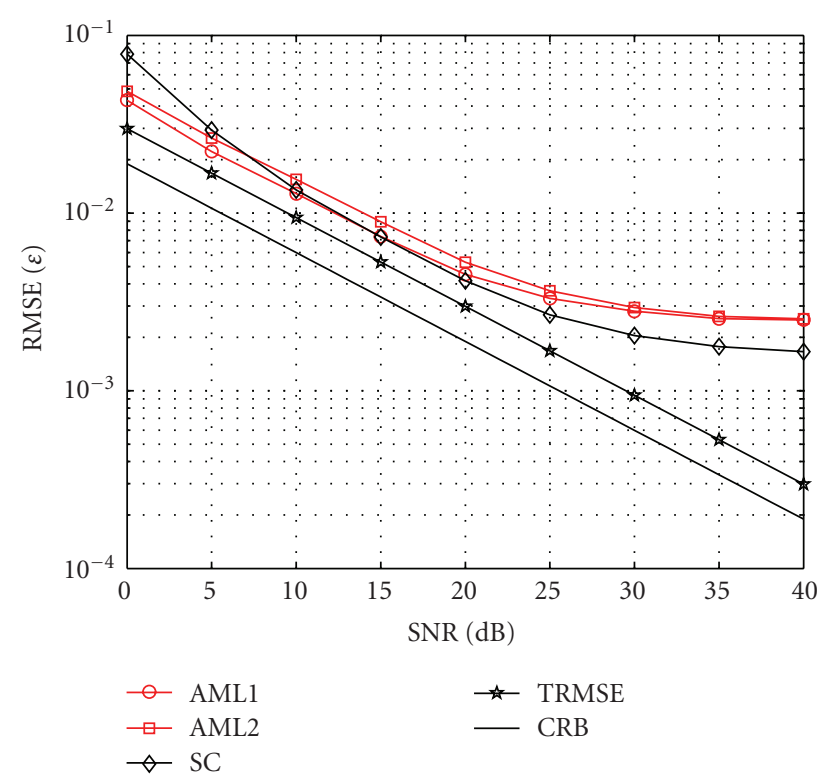

FIgure 4: Performance of the considered CFO estimators in ITU Vehicular A multipath channel.

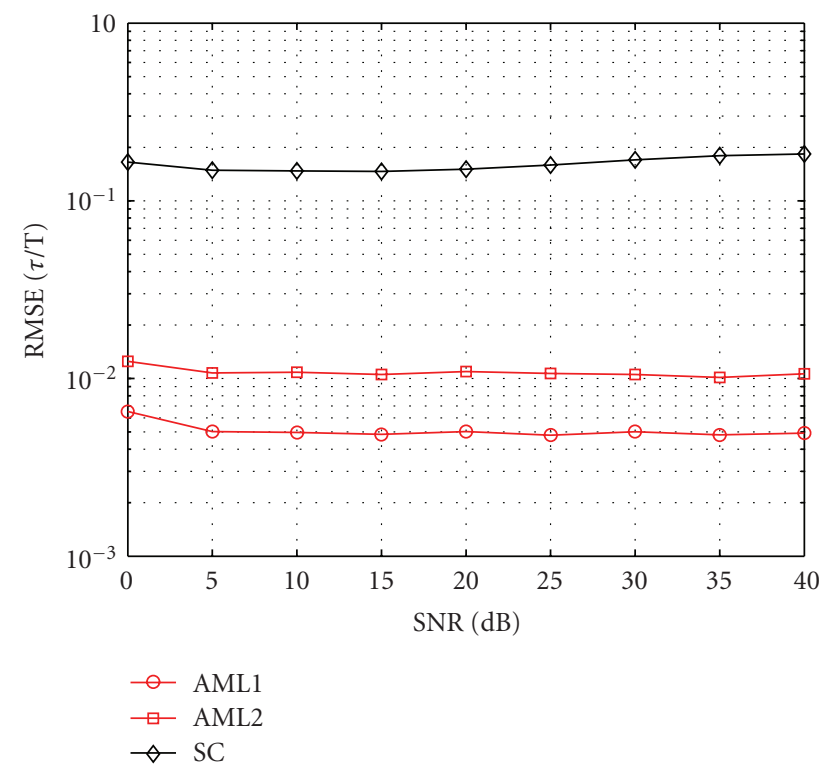

Figure 5: Performance of the considered ST estimators in ITU Vehicular A multipath channel.

that in AWGN the performance of both AML1 and AML2 estimators is coincident with that predicted by (29) for $\mathrm{SNR}=10 \mathrm{~dB}$ while is slightly different for SNR $=20 \mathrm{~dB}$. Moreover, Figure 8 shows that in multipath channel A the AML1 CFO estimator outperforms the AML2 estimator and assures estimates whose accuracy is quite similar to that provided by the SC estimator. As regards the performance of the AML and SC ST estimators results, not reported here for the sake of brevity, have shown that the SC ST estimator assures a normalized RMSE nearly equal to $10^{-1}$ both in AWGN and multipath channel due to the presence 


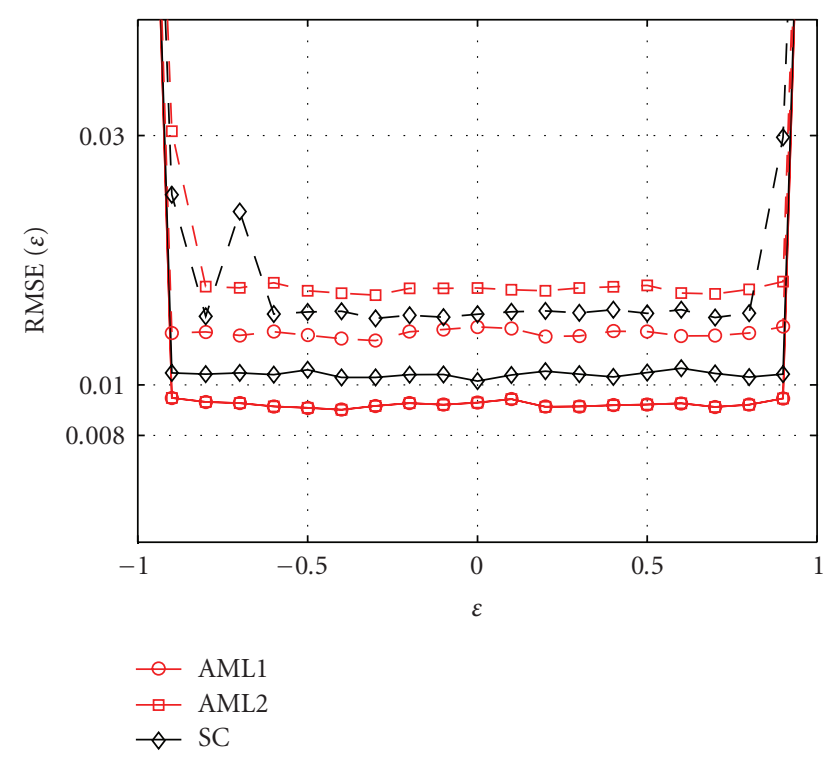

Figure 6: Performance of the considered CFO estimators as a function of the actual value of the normalized CFO.

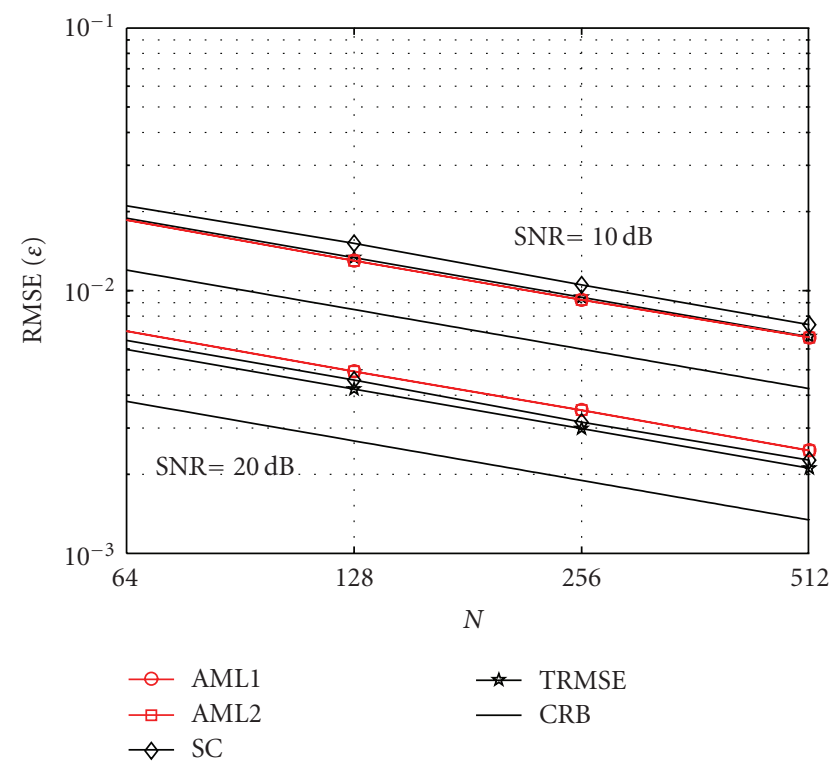

Figure 7: Performance of the considered CFO estimators as a function of the number of subcarriers in AWGN channel.

of the plateau. On the other hand, as regards the AML ST estimators no errors were observed in AWGN while a normalized RMSE less than $10^{-2}$ was obtained for $N \geq 64$.

Finally, Figures 9 and 10 show, for $N=256$ and $\varepsilon=$ 0.2 , the bit error rate (BER) obtained with the adoption of the AML and SC estimators followed by a one-tap equalizer with perfect knowledge of the channel and of the residual synchronization errors. The performance is compared with that of the perfectly synchronized OFDM/OQAM system (PS-OFDM/OQAM) and with that of the perfectly synchronized OFDM system $(\mathrm{PS}-\mathrm{OFDM})$ with $\mathrm{CP}=\mathrm{N} / 4$.

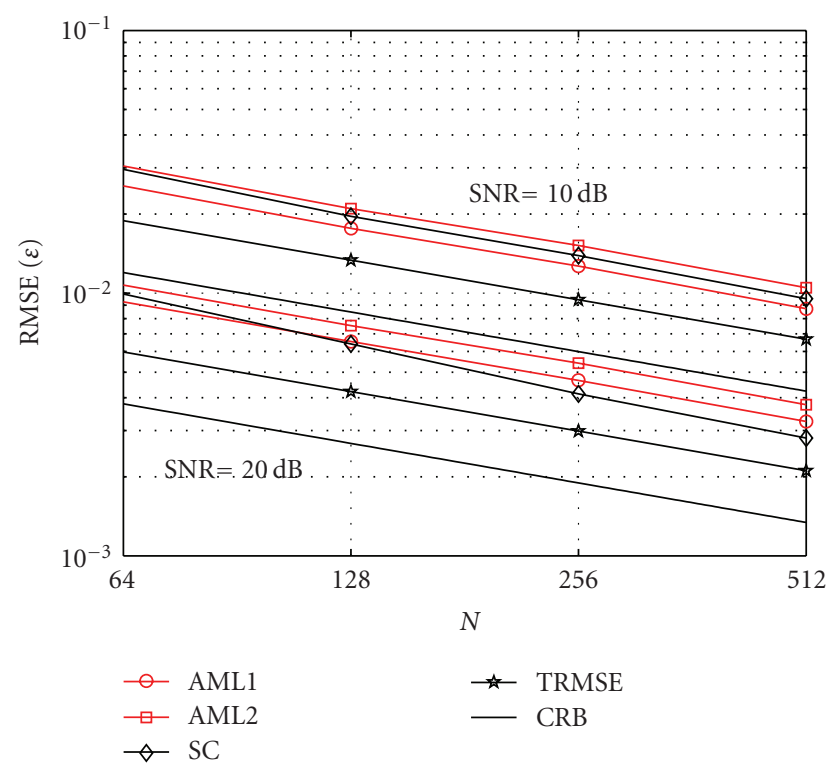

FIgURE 8: Performance of the considered CFO estimators as a function of the number of subcarriers in ITU Vehicular A multipath channel.

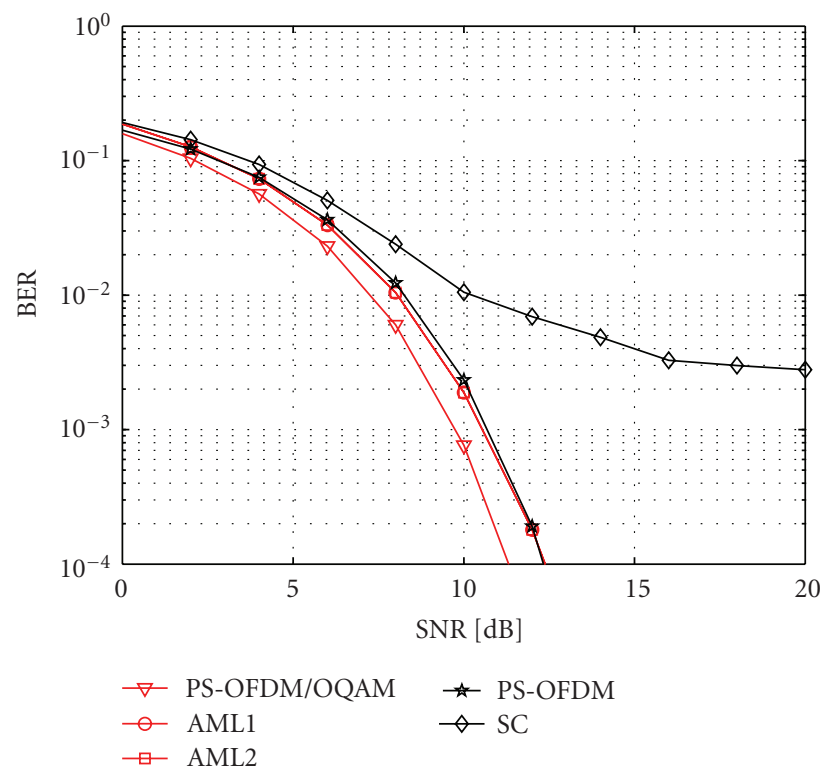

FIGURE 9: BER of the considered joint estimators in AWGN channel.

Note that the slight difference between the performance of the PS-OFDM/OQAM and that of the PS-OFDM is due to the fact that, to take into account the energy loss due to the $\mathrm{CP}$, the amplitude of the OFDM signal has been reduced by $\sqrt{1 /(1+\mathrm{CP} / N)}$ where $\mathrm{CP}=N / 4$. The results show that both in AWGN and multipath channel A the AML estimators assure a negligible degradation with respect to the perfectly synchronized system while the adoption of the SC synchronization scheme leads to an error floor due essentially to the inaccuracy of the ST estimates. 


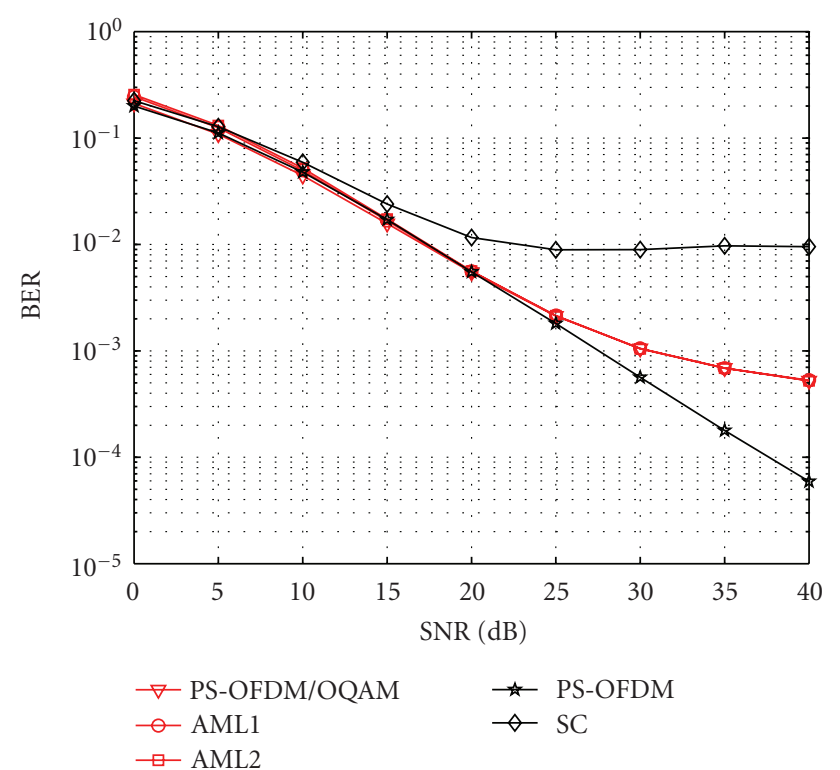

FIGURE 10: BER of the considered joint estimators in ITU Vehicular A multipath channel.

\section{Conclusions}

In this paper we have dealt with the problem of data-aided synchronization for OFDM/OQAM systems in multipath channels. In particular, the joint $\mathrm{ML}$ estimator for $\mathrm{CFO}$, amplitudes, phases, and delays, exploiting a short known preamble, has been derived. Exploiting the closed form ML estimators for phases and amplitudes and the closed form AML CFO estimator for small CFO values, a cost function that can provide an estimate of the ST, has been obtained. The performance of the joint AML1 estimator for multipath channel has been assessed via computer simulations and compared with that achieved by the joint AML2 estimator designed for AWGN channel. Moreover, a comparison with the performance achieved by the SC estimator for OFDM systems has been made. The results have shown that if it satisfied a condition involving the training symbol and the data burst is delayed by one OFDM/OQAM symbol interval with respect to the training burst, the AML CFO estimators assure a performance similar to that achieved by the SC estimator in multipath channel A, while the AML ST estimators outperform the SC estimator. Moreover, an approximate expression for the MSE of the AML CFO estimators has been derived that can be exploited to predict the actual performance in the range of moderate SNR values. Finally, a comparison between the BER obtained with the adoption of the AML and SC estimators followed by a one-tap equalizer with perfect knowledge of the channel and of the residual synchronization errors has been made. The results have shown that both in AWGN and multipath channel A the AML estimators assure a negligible degradation with respect to the perfectly synchronized system while the adoption of the SC synchronization scheme leads to an error floor due essentially to the inaccuracy of the ST estimates.

\section{Appendices}

\section{A.}

In this appendix we analyze the accuracy of the AML CFO estimates in the absence of noise in a single-path channel and in the case of perfect ST synchronization $\left(N_{c}=1\right.$ and $\tau_{1}=0$ in (1)). By considering a training symbol composed of $L=1$ OFDM/OQAM symbol, taking into account (18) and (20) it follows that

$$
\begin{aligned}
& w_{0}^{(l)}(0, \tilde{\mathcal{\varepsilon}}) \simeq \mathcal{u}_{l}(0)=\sum_{m=0}^{\beta N-1} r\left(m T_{s}\right) g\left(m T_{s}\right) e^{-j(2 \pi / N) m l} \\
& =\sqrt{\frac{N}{2 N_{u}}} \gamma e^{j \phi} \sum_{l_{1} \in \mathcal{P}} e^{j(\pi / 2) l_{1}} \\
& \times\left[a_{0, l_{1}}^{R} \sum_{m=0}^{\beta N-1} e^{j(2 \pi / N) \varepsilon m} \times g^{2}\left(m T_{s}\right) e^{-j(2 \pi / N) m\left(l-l_{1}\right)}\right. \\
& +j a_{0, l_{1}}^{I} \sum_{m=0}^{\beta N-1} e^{j(2 \pi / N) \varepsilon m} g\left(m T_{s}-\frac{T}{2}\right) \\
& \left.\times g\left(m T_{s}\right) e^{-j(2 \pi / N) m\left(l-l_{1}\right)}\right] \\
& =\sqrt{\frac{N}{2 N_{u}}} \gamma e^{j \phi} e^{j(\pi / 2) l} \\
& \times\left[a_{0, l}^{R} \sum_{m=0}^{\beta N-1} e^{j(2 \pi / N) \varepsilon m} g^{2}\left(m T_{s}\right)\right. \\
& \left.+j a_{0, l}^{I} \sum_{m=0}^{\beta N-1} e^{j(2 \pi / N) \varepsilon m} g\left(m T_{s}-\frac{T}{2}\right) g\left(m T_{s}\right)\right] \\
& +I_{w}(l, \varepsilon),
\end{aligned}
$$

where

$$
\begin{aligned}
I_{w}(l, \varepsilon) \triangleq & \sqrt{\frac{N}{2 N_{u}}} \gamma e^{j \phi} \sum_{l_{1} \in \mathcal{P}, l_{1} \neq l} e^{j(\pi / 2) l_{1}} \\
\times & {\left[a_{0, l_{1}}^{R} \sum_{m=0}^{\beta N-1} e^{j(2 \pi / N) \varepsilon m} g^{2}\left(m T_{s}\right) e^{-j(2 \pi / N) m\left(l-l_{1}\right)}\right.} \\
& +j a_{0, l_{1}}^{I} \sum_{m=0}^{\beta N-1} e^{j(2 \pi / N) \varepsilon m} g\left(m T_{s}-\frac{T}{2}\right) \\
& \left.\times g\left(m T_{s}\right) e^{-j(2 \pi / N) m\left(l-l_{1}\right)}\right] .
\end{aligned}
$$

Moreover, from (19) and (20) we have 


$$
\begin{aligned}
& \widehat{w}_{0}^{(l)}(0, \widetilde{\varepsilon}) \simeq e^{-j \pi \tilde{\varepsilon}} e^{-j \pi l} u_{l}\left(\frac{N}{2}\right) \\
& =e^{-j \pi \tilde{\varepsilon}} e^{-j \pi l} \sum_{m=0}^{\beta N-1} r\left[\left(m+\frac{N}{2}\right) T_{s}\right] g\left(m T_{s}\right) e^{-j(2 \pi / N) m l} \\
& =\sqrt{\frac{N}{2 N_{u}}} \gamma e^{j \phi} e^{-j \pi \tilde{\varepsilon}} e^{-j \pi l} \sum_{l_{1} \in \mathcal{P}} e^{j(3 \pi / 2) l_{1}} \\
& \times\left[a_{0, l_{1}}^{R} \sum_{m=0}^{\beta N-1} e^{j(2 \pi / N) \varepsilon(m+(N / 2))} g\left(m T_{s}+\frac{T}{2}\right)\right. \\
& \times g\left(m T_{s}\right) e^{-j(2 \pi / N) m\left(l-l_{1}\right)}+j a_{0, l_{1}}^{I} \\
& \left.\times \sum_{m=0}^{\beta N-1} e^{j(2 \pi / N) \varepsilon(m+(N / 2))} g^{2}\left(m T_{s}\right) e^{-j(2 \pi / N) m\left(l-l_{1}\right)}\right] \\
& =\sqrt{\frac{N}{2 N_{u}}} \gamma e^{j \phi} e^{-j \pi \tilde{\varepsilon}} e^{j(\pi / 2) l} e^{j \pi \varepsilon} \\
& \times\left[a_{0, l}^{R} \sum_{m=0}^{\beta N-1} e^{j(2 \pi / N) \varepsilon m} g\left(m T_{s}+\frac{T}{2}\right) g\left(m T_{s}\right)\right. \\
& \left.+j a_{0, l}^{I} \sum_{m=0}^{\beta N-1} e^{j(2 \pi / N) \varepsilon m} g^{2}\left(m T_{s}\right)\right] \\
& +e^{-j \pi \tilde{\varepsilon}} e^{-j \pi l} I_{\widehat{w}}(l, \varepsilon),
\end{aligned}
$$

where

$$
\begin{aligned}
I_{\widehat{w}}(l, \varepsilon) \triangleq & \sqrt{\frac{N}{2 N_{u}}} \gamma e^{j \phi} e^{j \pi \varepsilon} \sum_{l_{1} \in \mathcal{P}, l_{1} \neq l} e^{j(3 \pi / 2) l_{1}} \\
\times & {\left[a_{0, l_{1}}^{R} \sum_{m=0}^{\beta N-1} e^{j(2 \pi / N) \varepsilon m}\right.} \\
& \times g\left(m T_{s}+\frac{T}{2}\right) g\left(m T_{s}\right) e^{-j(2 \pi / N) m\left(l-l_{1}\right)} \\
& \left.+j a_{0, l_{1}}^{I} \sum_{m=0}^{\beta N-1} e^{j(2 \pi / N) \varepsilon m} g^{2}\left(m T_{s}\right) e^{-j(2 \pi / N) m\left(l-l_{1}\right)}\right] .
\end{aligned}
$$

Therefore, taking into account (18), (20), (22), and (A.1) it follows that

$$
\begin{aligned}
A(0)= & \sum_{l \in \mathcal{P}} e^{-j(\pi / 2) l} a_{0, l}^{R} w_{0}^{(l)}(0, \tilde{\mathcal{\varepsilon}})=\sqrt{\frac{N}{2 N_{u}}} \gamma e^{j \phi} \\
\times & {\left[\sum_{l \in \mathcal{P}}\left(a_{0, l}^{R}\right)^{2} \sum_{m=0}^{\beta N-1} e^{j(2 \pi / N) \varepsilon m} g^{2}\left(m T_{s}\right)\right.} \\
& \left.+j \sum_{l \in \mathcal{P}} a_{0, l}^{R} a_{0, l}^{I} \sum_{m=0}^{\beta N-1} e^{j(2 \pi / N) \varepsilon m} g\left(m T_{s}-\frac{T}{2}\right) g\left(m T_{s}\right)\right] \\
+ & \sum_{l \in \mathcal{P}} e^{-j(\pi / 2) l} a_{0, l}^{R} I_{w}(l, \varepsilon) .
\end{aligned}
$$

Moreover, from (19), (20), (23), and (A.3) we have

$$
\begin{aligned}
B(0)= & -j e^{j \pi \tilde{\varepsilon}} \sum_{l \in \mathcal{P}} e^{-j(\pi / 2) l} a_{0, l}^{I} \widehat{w}_{0}^{(l)}(0, \widetilde{\varepsilon}) \\
= & -j \sqrt{\frac{N}{2 N_{u}}} \gamma e^{j \phi} e^{j \pi \varepsilon} \\
\times & {\left[j \sum_{l \in \mathcal{P}}\left(a_{0, l}^{I}\right)^{2} \sum_{m=0}^{\beta N-1} e^{j(2 \pi / N) \varepsilon m} g^{2}\left(m T_{s}\right)\right.} \\
& \left.+\sum_{l \in \mathcal{P}} a_{0, l}^{R} a_{0, l}^{I} \sum_{m=0}^{\beta N-1} e^{j(2 \pi / N) \varepsilon m} g\left(m T_{s}+\frac{T}{2}\right) g\left(m T_{s}\right)\right] \\
& -j \sum_{l \in \mathcal{P}} e^{-j(3 \pi / 2) l} a_{0, l}^{I} I_{\widehat{w}}(l, \varepsilon) .
\end{aligned}
$$

Note that if the training symbol satisfies the condition

$$
\sum_{l \in \mathcal{P}} a_{0, l}^{R} a_{0, l}^{I}=0
$$

it follows that

$$
\begin{aligned}
A(0)= & \sqrt{\frac{N}{2 N_{u}}} \gamma e^{j \phi} \sum_{l \in \mathcal{P}}\left(a_{0, l}^{R}\right)^{2} \sum_{m=0}^{\beta N-1} e^{j(2 \pi / N) \varepsilon m} g^{2}\left(m T_{s}\right) \\
& +\sum_{l \in \mathcal{P}} e^{-j(\pi / 2) l} a_{0, l}^{R} I_{w}(l, \varepsilon), \\
B(0)= & e^{j \pi \varepsilon} \sqrt{\frac{N}{2 N_{u}}} \gamma e^{j \phi} \sum_{l \in \mathcal{P}}\left(a_{0, l}^{I}\right)^{2^{\beta N-1}} \sum_{m=0} e^{j(2 \pi / N) \varepsilon m} g^{2}\left(m T_{s}\right) \\
& +\sum_{l \in \mathcal{P}} e^{-j(\pi / 2)(1+3 l)} a_{0, l}^{I} I_{\widehat{w}}(l, \varepsilon) .
\end{aligned}
$$

In this case, if the interference terms $\sum_{l \in \mathcal{P}} e^{-j(\pi / 2) l} a_{0, l}^{R} I_{w}(l, \varepsilon)$ and $\sum_{l \in \mathcal{P}} e^{-j(\pi / 2)(1+3 l)} a_{0, l}^{I} I_{\widehat{w}}(l, \varepsilon)$ in the RHS of (A.8) and (A.9), respectively, were negligible, the $\mathrm{CFO}$ estimate would be (see (24) for $\left.N_{c}=1\right)(1 / \pi) \angle\left\{A^{*}(0) B(0)\right\}=\varepsilon$. The presence of the interference terms in the RHS of (A.8) and (A.9) leads to an error floor. Moreover, if condition (A.7) is not fulfilled an error floor can be observed also when the interference terms are negligible (see (A.5) and (A.6)). However, in the section on numerical results it is shown that the error floor can be substantially reduced when condition (A.7) is satisfied.

\section{B.}

In this appendix we derive an approximate expression for the MSE of the AML CFO estimator reported in (24) for a singlepath channel and in the case of perfect ST synchronization $\left(N_{c}=1\right.$ and $\tau_{1}=0$ in (1)). Specifically, we consider the case where the SNR is such that interference terms in (A.8) and (A.9) can be neglected with respect to the noise terms due to AWGN but it is sufficiently high that noise $\times$ noise terms 
can be deleted. In this case taking into account (A.1), (A.3), (A.8), and (A.9) it follows that

$$
\begin{aligned}
A^{*}(0) B(0) & \simeq\left[A_{0}^{*}(0)+W_{A}^{*}\right]\left[B_{0}(0)+W_{B}\right], \\
& \simeq A_{0}^{*}(0) B_{0}(0)+A_{0}^{*}(0) W_{B}+B_{0}(0) W_{A}^{*},
\end{aligned}
$$

where the last approximation has been obtained by neglecting the noise $\times$ noise term, and, moreover, $A_{0}(0)$ and $B_{0}(0)$ represent the contribution to $A(0)$ and $B(0)$ (in (A.8) and (A.9), resp.) in the absence of noise and of the interference terms. The noise contributions in (B.1) are given by

$$
\begin{aligned}
W_{A} \triangleq & \sum_{k=0}^{\beta N-1} n\left(k T_{s}\right) g\left(k T_{s}\right) \sum_{l \in \mathcal{P}} a_{0, R}^{I} e^{-j(\pi / 2) l} e^{-j(2 \pi / N) k l}, \\
W_{B} \triangleq & -j \sum_{k=0}^{\beta N-1} n\left[\left(k+\frac{N}{2}\right) T_{s}\right] \\
& \times g\left(k T_{s}\right) \sum_{l \in \mathcal{P}} a_{0, I}^{I} e^{-j(\pi / 2) l} e^{-j \pi l} e^{-j(2 \pi / N) k l}
\end{aligned}
$$

where the zero-mean circular noise $n\left(k T_{s}\right)$ has a variance $\mathrm{E}\left[\left|n\left(k T_{s}\right)\right|^{2}\right]=\sigma_{n}^{2} / T_{s}$. In this case we obtain

$$
\begin{aligned}
\hat{\varepsilon}-\varepsilon & =\frac{1}{\pi} \angle\left\{A^{*}(0) B(0) e^{-j \pi \varepsilon}\right\} \\
& \simeq \frac{1}{\pi} \frac{\mathfrak{I}\left\{e^{-j \pi \varepsilon} A_{0}^{*}(0) W_{B}+e^{-j \pi \varepsilon} B_{0}(0) W_{A}^{*}\right\}}{\mathfrak{R}\left\{A_{0}^{*}(0) B_{0}(0)\right\}} \\
& =\frac{1}{\pi} \frac{\mathfrak{I}\{Z\}}{\left(N / 2 N_{u}\right) \gamma^{2} \sum_{l \in \mathcal{P}}\left(a_{0, l}^{R}\right)^{2} \sum_{l \in \mathcal{P}}\left(a_{0, l}^{I}\right)^{2} \mathfrak{q}} \\
& =\frac{1}{\pi} \frac{\mathfrak{I}\{Z\}}{(N / 2) \gamma^{2} N_{u}\left|\sum_{k=0}^{\beta N-1} e^{j(2 \pi / N) \varepsilon k} g^{2}\left(k T_{s}\right)\right|^{2}},
\end{aligned}
$$

where $\mathfrak{q}$ denotes $\left|\sum_{k=0}^{\beta N-1} e^{j(2 \pi / N) \varepsilon k} g^{2}\left(k T_{s}\right)\right|^{2}$ and the last equality has been obtained by exploiting the fact that $a_{p, l}^{R}$, $a_{p, l}^{I} \in\{-1,1\}$, and, moreover,

$$
Z \triangleq e^{-j \pi \varepsilon} A_{0}^{*}(0) W_{B}+e^{-j \pi \varepsilon} B_{0}(0) W_{A}^{*}
$$

Thus, from (B.4) we obtain

$$
E\left[(\hat{\varepsilon}-\varepsilon)^{2}\right] \simeq \frac{1}{2 \pi^{2}} \frac{E\left[|Z|^{2}\right]-\mathfrak{R}\left\{E\left[Z^{2}\right]\right\}}{\left[(N / 2) \gamma^{2} N_{u}\left|\sum_{k=0}^{\beta N-1} e^{j(2 \pi / N) \varepsilon k} g^{2}\left(k T_{s}\right)\right|^{2}\right]^{2}}
$$

Finally, under the assumption $\sum_{l \in \mathcal{P}} a_{0, l}^{R} a_{0, l \pm 1}^{I} \simeq 0$, exploiting the condition (A.7) and the noise circularity, we have

$$
\begin{gathered}
\mathfrak{R}\left\{E\left[Z^{2}\right]\right\} \simeq 0, \\
E\left[|\mathrm{Z}|^{2}\right]=\frac{\sigma_{n}^{2}}{T_{s}} \sum_{k=0}^{\beta N-1} g^{2}\left(k T_{s}\right) N \gamma^{2} N_{u}^{2}\left|\sum_{k=0}^{\beta N-1} e^{j(2 \pi / N) \varepsilon k} g^{2}\left(k T_{s}\right)\right|^{2} .
\end{gathered}
$$

Therefore, we can write

$$
E\left[(\widehat{\varepsilon}-\varepsilon)^{2}\right] \simeq \frac{2}{\pi^{2} N S N R} \frac{1}{\left|\sum_{k=0}^{\beta N-1} \bar{g}(k)^{2} e^{j(2 \pi / N) \varepsilon k}\right|^{2}},
$$

where SNR $\triangleq \gamma^{2} / \sigma_{n}^{2}$ and $\bar{g}(k) \triangleq g\left(k T_{s}\right) \sqrt{\sum_{l=0}^{\beta N-1} g^{2}\left(l T_{s}\right)}$.

\section{Acknowledgment}

This work was supported in part by the European Commission under Project PHYDYAS (FP7-ICT-2007-1-211887).

\section{References}

[1] B. Farhang-Boroujeny and R. Kempter, "Multicarrier communication techniques for spectrum sensing and communication in cognitive radios," IEEE Communications Magazine, vol. 46, no. 4, pp. 80-85, 2008.

[2] G. Cherubini, E. Eleftheriou, S. Oker, and J. M. Cioffi, "Filter bank modulation techniques for very high-speed digital subscriber lines," IEEE Communications Magazine, vol. 38, no. 5, pp. 98-104, 2000.

[3] T. Ihalainen, T. H. Stitz, M. Rinne, and M. Renfors, "Channel equalization in filter bank based multicarrier modulation for wireless communications," EURASIP Journal on Advances in Signal Processing, vol. 2007, Article ID 49389, 18 pages, 2007.

[4] D. Lacroix, N. Goudard, and M. Alard, "OFDM with guard interval versus OFDM/OffsetQAM for high data rate UMTS downlink transmission," in Proceedings of the 54th IEEE Vehicular Technology Conference (VTC '01), vol. 4, pp. 26822686, Atlantic City, NJ, USA, October 2001.

[5] C. R. M. Alard and P. Siohan, "A family of extended gaussian functions with a nearly optimal localization," in Proceedings of the 1st International Workshop on Multi-Carrier SpreadSpectrum, pp. 179-186, Oberpfaffenhofen, Germany, April 1997.

[6] R. Haas and J.-C. Belfiore, "A time-frequency well-localized pulse for multiple carrier transmission," Wireless Personal Communications, vol. 5, no. 1, pp. 1-18, 1997.

[7] P. Ciblat and E. Serpedin, "A fine blind frequency offset estimator for OFDM/OQAM systems," IEEE Transactions on Signal Processing, vol. 52, no. 1, pp. 291-296, 2004.

[8] T. Fusco and M. Tanda, "Blind frequency-offset estimation for OFDM/OQAM systems," IEEE Transactions on Signal Processing, vol. 55, no. 5, pp. 1828-1838, 2007.

[9] H. Bolcskei, "Blind estimation of symbol timing and carrier frequency offset in wireless OFDM systems," IEEE Transactions on Communications, vol. 49, no. 6, pp. 988-999, 2001.

[10] T. Fusco, A. Petrella, and M. Tanda, "Data-aided symbol timing and cfo synchronization for filter-bank multicarrier systems," IEEE Transactions on Wireless Communications, vol. 8, no. 5, pp. 2705-2715, 2009. 
[11] B. Jahan, M. Lanoiselée, G. Degoulet, and R. Rabineau, "Full synchronization method for OFDM/OQAM and OFDM/QAM modulations," in Proceedings of the 10th IEEE International Symposium on Spread Spectrum Techniques and Applications (ISSSTA '08), pp. 344-348, August 2008.

[12] B. Jahan, M. Lanoiselée, G. Degoulet, and R. Rabineau, "Frame synchronization method for OFDM/QAM and ODFM/OQAM modulations," in Proceedings of the 4th IEEE International Conference on Circuits and Systems for Communications (ICCSC '08), pp. 445-449, Shanghai, China, May 2008.

[13] T. Fusco, A. Petrella, and M. Tanda, "Joint symbol timing and CFO estimation in multiuser OFDM/OQAM systems," in Proceedings of the 10th IEEE Workshop on Signal Processing Advances in Wireless Communications (SPAWC '09), pp. 613617, Perugia, Italy, June 2009.

[14] T. M. Schmidl and D. C. Cox, "Robust frequency and timing synchronization for OFDM," IEEE Transactions on Communications, vol. 45, no. 12, pp. 1613-1621, 1997.

[15] M. G. Bellanger, "Specification and design of a prototype filter for filter bank based multicarrier transmission," in Proceedings of the IEEE International Conference on Acoustics, Speech, and Signal Processing (ICASSP '01), vol. 4, pp. 2417-2420, Salt Lake, Utah, USA, May 2001.

[16] Recommendation ITU-R M. 1225, "Guidelines for evaluation of radio transmission technologies for IMT-2000," 1997. 